\title{
Variation in contrast-associated acute kidney injury prophylaxis for percutaneous coronary intervention: insights from the Veterans Affairs Clinical Assessment, Reporting, and Tracking (CART) program
}

\author{
Joseph Walker Keach ${ }^{1,2^{*}}$, Maggie A. Stanislawski, ${ }^{3,4}$ Anna E. Barón ${ }^{3,4}$, Mary E. Plomondon ${ }^{3,4}$, Paula Langner ${ }^{3}$,
} Amit Amin ${ }^{5}$, Heather M. Gilmartin ${ }^{3,4}$, Stephen Waldo ${ }^{2,3}$ and Thomas M. Maddox 5,6

\begin{abstract}
Background: Contrast-Associated Acute Kidney Injury (CA-AKI) is a serious complication associated with percutaneous coronary intervention (PCI). Patients with chronic kidney disease (CKD) have an elevated risk for developing this complication. Although CA-AKI prophylactic measures are available, the supporting literature is variable and inconsistent for periprocedural hydration and $\mathrm{N}$-acetylcysteine (NAC), but is stronger for contrast minimization.

Methods: We assessed the prevalence and variability of CA-AKI prophylaxis among CKD patients undergoing PCI between October 2007 and September 2015 in any cardiac catheterization laboratory in the VA Healthcare System. Prophylaxis included periprocedural hydration with normal saline or sodium bicarbonate, NAC, and contrast minimization (contrast volume to glomerular filtration rate ratio $\leq 3$ ). Multivariable hierarchical logistic regression models quantified site-specific prophylaxis variability. As secondary analyses, we also assessed CA-AKI prophylaxis measures in all PCI patients regardless of kidney function, periprocedural hydration in patients with comorbid CHF, and temporal trends in CA-AKI prophylaxis.

\footnotetext{
*Correspondence: walker.keach.md@gmail.com; joseph.keach@dhha.org 'Department of Medicine, Denver Health Hospital Authority, 601 Broadway MC4000, Denver, CO 80204, USA

${ }^{2}$ Department of Medicine, University of Colorado, Aurora, CO, USA

Full list of author information is available at the end of the article
}

(c) The Author(s). 2020 Open Access This article is licensed under a Creative Commons Attribution 4.0 International License, which permits use, sharing, adaptation, distribution and reproduction in any medium or format, as long as you give appropriate credit to the original author(s) and the source, provide a link to the Creative Commons licence, and indicate if changes were made. The images or other third party material in this article are included in the article's Creative Commons licence, unless indicated otherwise in a credit line to the material. If material is not included in the article's Creative Commons licence and your intended use is not permitted by statutory regulation or exceeds the permitted use, you will need to obtain permission directly from the copyright holder. To view a copy of this licence, visit http://creativecommons.org/licenses/by/4.0/ The Creative Commons Public Domain Dedication waiver (http://creativecommons.org/publicdomain/zero/1.0/) applies to the data made available in this article, unless otherwise stated in a credit line to the data. 


\begin{abstract}
(Continued from previous page)
Results: From 2007 to 2015, 15,729 patients with CKD underwent PCI. 6928 (44.0\%) received periprocedural hydration (practice-level median rate 45.3\%, interquartile range (IQR) 35.5-56.7), 5107 (32.5\%) received NAC (practice-level median rate 28.3\%, IQR 22.8-36.9), and 4656 (36.0\%) received contrast minimization (practice-level median rate 34.5, IQR 22.6-53.9). After adjustment for patient characteristics, there was significant site variability with a median odds ratio (MOR) of 1.80 (Cl 1.56-2.08) for periprocedural hydration, 1.95 (Cl 1.66-2.29) for periprocedural hydration or NAC, and 2.68 (Cl 2.23-3.15) for contrast minimization. These trends were similar among all patients (with and without CKD) undergoing PCl. Among patients with comorbid CHF $(n=5893), 2629$ (44.6\%) received periprocedural hydration, and overall had less variability in hydration (MOR of 1.56 (Cl 1.38-1.76)) compared to patients without comorbid CHF (1.89 (Cl 1.65-2.18)). Temporal trend analysis showed a significant and clinically relevant decrease in NAC use (64.1\% of cases in $2008(N=1059), 6.2 \%$ of cases in $2015(N=128, p=<$ $0.0001)$ ) and no significant change in contrast-minimization ( $p=0.3907)$.

Conclusions: Among patients with CKD undergoing PCl, there was low utilization and significant site-level variability for periprocedural hydration and NAC independent of patient-specific risk. This low utilization and high variability, however, was also present for contrast minimization, a well-established measure. These findings suggest that a standardized approach to CA-AKI prophylaxis, along with continued development of the evidence base, is needed.
\end{abstract}

Keywords: Prevention, AKI, CA-AKI, PCI, CKD

\section{Relationships with industry}

Dr. Waldo receives research support to the Denver Research Institute from Abiomed, Cardiovascular Systems Incorporated and Merck Pharmaceuticals. Dr. Maddox discloses current grant funding from the NIH NCATS (1U24TR002306-01, A National Center for Digital Health Informatics Innovation), current consulting for Creative Educational Concepts, Inc. and Atheneum Partners, and honoraria payments in the past 3 years from Brown University (Sept 2016), Washington State Clinical Outcomes Assessment Program (Oct 2016), Virginia Mason (Oct 2016), University of Utah (May 2017), New York Presbyterian (Sept 2017), Westchester Medical Center (Oct 2017), Sentara Heart Hospital (Dec 2018), and the Henry Ford health system (March 2019). He is currently employed as a cardiologist and director of the Healthcare Innovation Lab at BJC HealthCare/Washington University School of Medicine. In this capacity, he is advising Myia Labs, for which his employer is receiving equity compensation in the company. He is receiving no individual compensation from the company. $\mathrm{He}$ is also a compensated director for a New Mexico-based foundation, the J. F Maddox Foundation.

The other authors have no industry relations to disclose.

\section{Background}

Contrast-Associated Acute Kidney Injury (CA-AKI), defined as an absolute increase of serum creatinine $\geq 0.3$ $\mathrm{mg} / \mathrm{dL}$ or a relative increase $\geq 50 \%$ within $48-72 \mathrm{~h}$ of contrast exposure, is a serious complication that may occur after percutaneous coronary intervention (PCI) [1]. CA-AKI is associated with increased morbidity, mortality, hospital length of stay, and healthcare costs [2-5].
Chronic kidney disease (CKD) is the strongest independent risk factor for developing CA-AKI [6]. Unfortunately, effective therapies to reduce the clinical impact of CAAKI among CKD patients are largely absent. Thus, the primary therapeutic objective is to reduce the likelihood of developing CA-AKI among these patients. Numerous prophylactic interventions have been studied, including periprocedural intravenous hydration with normal saline (NS) or sodium bicarbonate, periprocedural administration of N-Acetylcysteine (NAC), and the minimization of contrast volume [7-11]. The strength of evidence supporting NAC and specific periprocedural hydration protocols is weak, while minimizing procedural contrast volume is much stronger [7-11]. Major society guidelines on CA-AKI prophylaxis in CKD patients are currently lacking or contradictory [12, 13]. We hypothesized that such variability in the literature and guidelines would result in significant site-level practice variation independent of patient-specific factors. We also hypothesized there would be less variability in well-established interventions, such as contrast minimization. With this in mind, we sought to assess the prevalence and variation of CA-AKI prophylactic measures utilized in CKD patients undergoing PCI throughout the largest integrated healthcare system in the United States, the Veterans Affairs (VA) Healthcare System, with hopes of identifying opportunities to improve the use of prophylactic measures and reduce the incidence of CA-AKI.

\section{Methods}

The VA CART program is a national clinical quality initiative for all VA cardiac catheterization laboratories. The program seeks to enhance the quality and safety of 
invasive cardiovascular procedures throughout the VA [14]. The CART program collects standardized data on all coronary angiograms and percutaneous coronary interventions. Software is embedded in the VA electronic health record (EHR) and allows providers to enter patient and procedural information as part of routine clinical workflow. The CART software was designed using standardized definitions which conform to the definitions and standards of the American College of Cardiology's National Cardiovascular Data Registry (ACC-NCDR) [15]. Quality checks of the data are periodically conducted for completeness and accuracy [16]. CART data are combined with other VA data sources to create a longitudinal data repository that supports quality assessment and improvement across the integrated healthcare system.

We evaluated the use of periprocedural hydration with normal saline or sodium bicarbonate, periprocedural $\mathrm{NAC}$, and the volume of contrast used during PCI relative to the patient's GFR among patients with CKD undergoing PCI between October 1st 2007 and September 30th 2015 at any VA cardiac catheterization laboratory. Prior studies have indicated that ratios of contrast volume relative to GFR $<3$ are associated with lower CA-AKI risk [10]. CKD was defined as a glomerular filtration rate (GFR) of $15-59 \mathrm{~mL} / \mathrm{min}$. The use of periprocedural hydration was determined from in-lab records and/or inpatient medications within $48 \mathrm{~h}$ of the procedure and included the administration of normal saline (NS) or intravenous sodium bicarbonate. Doses of periprocedural hydration $<100 \mathrm{~mL}$ were excluded to avoid mistaking saline flushes and other small saline applications for CA-AKI pre-hydration. The median volume of preprocedural hydration administered using this approach was $1000 \mathrm{ml}$ (IQR: $500-1000 \mathrm{ml}$ ). The use of NAC was determined from in-lab records and/or outpatient prescription data, including prescriptions for $\leq 3$ days of NAC given within 30 days of the procedure. The PCI providers recorded contrast volume administration in the EHR. GFR levels were calculated using the Modification of Diet in Renal Disease (MDRD) equation, using the most recent serum creatinine value recorded within 30 days of the PCI [17]. Patients were excluded for currently receiving hemodialysis, missing preprocedural GFR assessment, missing longitudinal followup data, undergoing repeat PCI, or receiving PCI at a facility that performed $<50$ PCIs over the time frame of the study.

We assessed patient demographic and clinical risk factors, including age, race (white, black, other), sex, presence of congestive heart failure (CHF), GFR, and history of diabetes, from the EHR using standard definitions [15]. Lesion risk (defined as high or non-high) and number of stents were determined by data entered into the
CART application by physicians. Annual PCI volume was assessed using CART data.

We compared demographic, clinical, and CA-AKI prophylaxis characteristics of CKD patients undergoing PCI by catheterization laboratory rates of periprocedural hydration or NAC use, stratified into quartiles. We used chi-squared tests for categorical variables and MannWhitney Wilcoxon tests for continuous variables. We then calculated unadjusted rates of 1) Periprocedural hydration, 2) NAC use, 3) periprocedural hydration or NAC use, and 4) contrast: GFR $\leq 3$. Median site-level rates with interquartile ranges were calculated.

Next, to evaluate the variation in use of site-level CA-AKI prophylaxis measures independent of patient CA-AKI risk, we calculated adjusted rates of 1 ) periprocedural hydration, 2) periprocedural hydration or NAC, and 3) contrast: GFR $\leq 3$. We did not model the outcome of NAC due to instability in statistical models caused by the wide variation in NAC use across sites with very small numbers at many sites. For the analysis of each prophylaxis measure, catheterization laboratories were excluded if the prophylactic measure was used infrequently ( $<20$ times over the study period) to avoid unstable estimates. For the contrast: GFR ratio analyses, PCI patients who had missing contrast volume information were excluded.

We used hierarchical regression models with VA catheterization laboratory sites as a random intercept to adjust for differences in lab case mix and to account for the clustering of patients by lab, similar to the methodology used in calculating hospital-level readmission data by the Centers for Medicare and Medicaid [18]. Based on prior literature and a priori clinical knowledge, we adjusted for the following patient level characteristics associated with CA-AKI: age, race, sex, CHF, GFR, diabetes, lesion risk (at least 1 high risk vs all non-high risk), number of stents, and year of PCI, as well as sitelevel annual PCI volume. Lesion risk and number of stents were included as surrogates for PCI complexity. For binary outcomes, we used logistic regression models to calculate the predicted and expected probability of each prevention measure at each lab and then multiplied this ratio by the mean rate across sites to get the standardized site-level rate [19]. We estimated the 95\% confidence interval using bootstrap sampling. We considered the lab proportion as significantly different from average when the $95 \%$ confidence interval did not include the system-wide mean.

We used the median odds ratio (MOR) to describe the level of variation between sites, independent of patient factors. The MOR can be interpreted as the odds that 2 patients with identical patient-level covariates from 2 randomly chosen sites will receive similar treatments, or in our case, prophylaxis. An MOR of 1.0 indicates that 
no variation exists between sites, and identical patients would receive identical treatment at different sites. The MOR is always $\geq 1$. For example, an MOR of 1.5 indicates a $50 \%$ likelihood that a similar patient would receive different prophylactic measures at 2 different sites. It provides an estimate of the effect of the catheterization laboratory on the outcome, much as the odds ratio (OR) estimates the effect of patient factors on the outcome. Based on previous literature, an MOR > 1.2 indicates moderate site-level variation [20].

We also performed several secondary analyses. First, we expanded the cohort to all patients undergoing PCI, regardless of kidney function. Methods for this analysis of all patients were identical to those used for the primary analysis.

Second, to evaluate the potential effect of comorbid $\mathrm{CHF}$ on the volume of IV fluid administered for prophylaxis, we stratified the cohort by presence of CHF. We then performed hierarchical regression models of periprocedural hydration, identical to those used for the primary analysis, to estimate use, and variation in use, within each stratum.
Finally, to explore the temporal changes in prophylaxis, a linear trend test was applied to unadjusted rates of use of each measure by fiscal year.

All statistical analyses were performed by the CART Program Analytic Center using SAS version 9.4 (SAS Institute Inc., Cary, North Carolina) and R 3.4.1 (R Core Team (2019). The Colorado Multiple Institutional Review Board approved the study.

\section{Results}

Between 2007 and 2015, 87,056 PCIs were performed across the VA integrated healthcare system. As shown in Fig. 1, PCIs were excluded if patients were currently receiving hemodialysis $(N=2727)$, missing pre-procedural GFR assessment $(N=4567)$, without longitudinal followup data $(N=2)$, undergoing repeat PCI $(N=15,588)$, sites with $<50$ PCIs over the time frame of the study $(N=37)$, and or had normal kidney function $(N=48,406)$. Thus, the cohort for our primary analysis included 15,729 patients with CKD undergoing PCI at 64 unique sites.

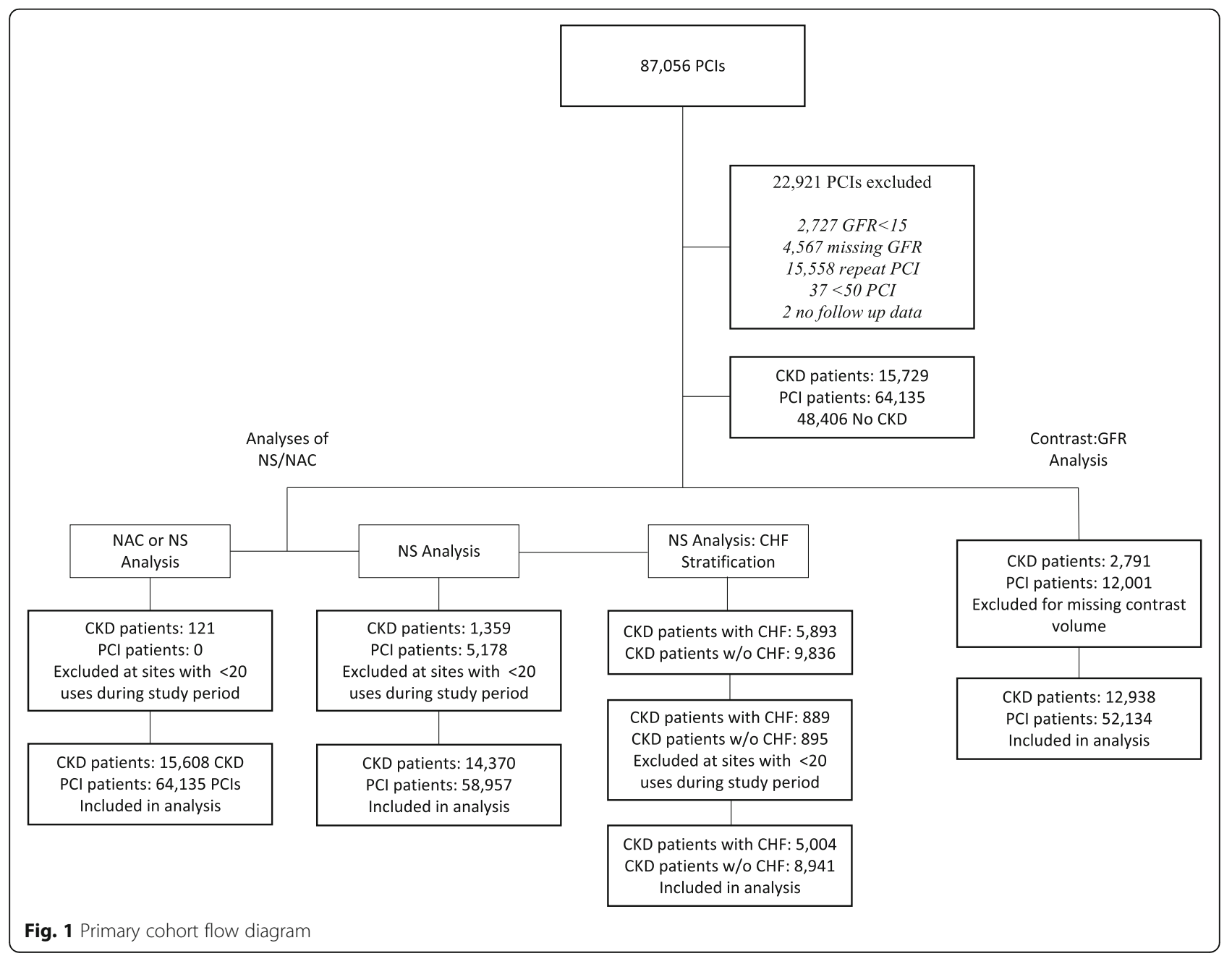


Table 1 shows the characteristics of the primary CKD cohort, both overall and arranged by quartile of increasing unadjusted rates of periprocedural hydration or NAC use. Among these 15,729 patients, 6928 (44.0\%) received periprocedural hydration (practice-level median rate $45.3 \%$, interquartile range (IQR) 35.5-56.7), 5107 (32.5\%) received NAC (practice-level median rate 28.3\%, IQR 22.8-36.9), and 9436 (60.0\%) received periprocedural hydration or NAC (practice-level median rate 62.8, IQR 50.7-72.4). The median contrast: GFR ratio was 3.6, (IQR 2.5-5.1) and 4656 (36.0\%) patients received contrast minimization (contrast: GFR ratio $\leq 3$, practice-level median rate 34.5, IQR 22.6-53.9). After adjusting for patient-specific risk factors, the MOR for only periprocedural hydration was 1.80 (CI 1.59-2.08), for periprocedural hydration or NAC was 1.95 (confidence interval [CI] 1.66-2.29), and contrast: GFR ratio $\leq 3$ was 2.68 (CI 2.23-3.15) (Table 2; Figs. 2 and 3). The intracluster correlation coefficient for periprocedural hydration was 0.07 , for hydration or NAC, 0.13 , and for contrast minimization, 0.18 .

For our secondary analysis of all PCI patients, we included all patients undergoing PCI during the study period regardless of kidney function. Among this cohort of 64,135 PCI patients, 22,888 (35.7\%) received periprocedural hydration, 8919 (13.9\%) received NAC, and $27,595(43 \%)$ received periprocedural hydration or NAC. The median contrast volume to GFR ratio was 2.5 (IQR 1.6-3.7) and 32,503 (62.3\%) patients had a contrast: GFR ratio $<=3$. After adjustment, the MOR for periprocedural hydration was 1.96 (CI 1.71-2.24), and for periprocedural hydration or NAC use was 2.15 (CI 1.82-2.53). The MOR for a contrast: GFR ratio $\leq 3$ was 2.75 (CI 2.26-3.28) (Table 2; Supplemental Figs. 1 and 2).

For our secondary analysis of comorbid CHF and periprocedural hydration, 5893 (37.5\%) patients from our primary cohort had CHF (9836 had CKD but not CHF). 2629 (44.61\%) patients with CHF received periprocedural hydration, while 4299 (43.71\%) of those without CHF received hydration. After adjusting for patientspecific risk factors, the MOR for any periprocedural hydration among patients with CHF was 1.56 (1.38-1.76). For those without CHF, the MOR was 1.89 (1.65-2.18) (Supplemental Fig. 3).

Finally, for our secondary analysis of temporal prophylaxis trends, there was a significant and clinically relevant decrease in NAC use over the study period, with NAC being used in $64.1 \%$ of cases in $2008(N=12,059)$, and only $6.2 \%$ of cases in 2015 $(N=128, p=<0.0001)$ (Supplemental Tables 1a, b, c). Contrast minimization appeared to stay constant with no significant trend change over the study period $(p=0.3907)$.

\section{Discussion}

Our analysis of CA-AKI prophylaxis among patients with CKD undergoing PCI in the national VA healthcare system demonstrated low utilization of commonly employed prophylactic measures, as well as a high level of variability in practice patterns, independent of patient-specific risk. This low utilization and high variability were present for well-established, as well as less established, prophylactic measures and similar trends were noted in PCI patients with and without CKD. We expected there would be less variability in CA-AKI prophylaxis in the highest risk population, patients with CKD, compared to the general PCI population. We found, however, that high levels of variability persisted among CKD patients, with contrast minimization even more variable in this population.

Among patients with CKD, periprocedural hydration and NAC, both poorly-established interventions, were administered in a little over half of procedures, and had an MOR of 1.80, indicating that identical patients would have roughly 2 -fold greater odds of receiving hydration or NAC by being treated in one facility compared to another. However, the well-established measure of contrast minimization had even higher variability by site (MOR 2.68). This demonstrates that identical patients with CKD have almost 3-fold greater odds of receiving high volumes of contrast in 1 cardiac catheterization laboratory compared to another.

Among patients with comorbid CHF, we found that patients with CKD and comorbid CHF received periprocedural hydration at a similar frequency $(44.61 \%$ vs 43.71\%). Furthermore, after risk adjustment, there was less variability in periprocedural hydration of patients with CHF than those without (MOR 1.56 vs 1.89 , respectively). This is likely related to provider preference and avoidance of potential volume overload in patients at high risk for this complication (patients with impaired cardiac and renal function).

Our study evaluated 8 years of CA-AKI prophylaxis practice patterns. During this time significant research was published and subsequent guidelines updated. We observed a statistically significant and clinically relevant decrease in the use of NAC prophylaxis over the time course of our study, falling from $64.1 \%$ of cases in 2008 to only $6.2 \%$ in 2015 . This is likely related to highquality research demonstrating the lack of benefit of NAC for CA-AKI prophylaxis, and the updated 2011 ACC/AHA/SCAI guidelines recommended against the use of NAC [12]. Of note, the well-established prophylactic measure of contrast-minimization remained relatively low and constant throughout our study period. Contrast-minimization was on average utilized only $36.0 \%$ of the time and ranged from 33.6 to $38.9 \%$ with no significant increase in utilization over time. 
Table 1 Demographic and clinical characteristics of patients with CKD receiving PCI arranged by site quartile of unadjusted rates of periprocedural hydration or NAC use

\begin{tabular}{|c|c|c|c|c|c|c|}
\hline Variable & $\begin{array}{l}\text { Total } \\
(N=15,729)\end{array}$ & $\begin{array}{l}\text { QUARTILE } 1 \\
(N=3507)\end{array}$ & $\begin{array}{l}\text { QUARTILE } 2 \\
(N=4108)\end{array}$ & $\begin{array}{l}\text { QUARTILE } 3 \\
(N=4511)\end{array}$ & $\begin{array}{l}\text { QUARTILE } 4 \\
(N=3603)\end{array}$ & $p$-value \\
\hline \multicolumn{7}{|l|}{ Demographics } \\
\hline Age (Median (IQR)) & $68.6(63.6-76.9)$ & $68.8(63.5-76.8)$ & $67.7(63.1-75.6)$ & $68.6(63.4-76.9)$ & $70.0(64.3-78.7)$ & $<.0001$ \\
\hline Male & $15,429(98.1 \%)$ & $3439(98.1 \%)$ & $4022(97.9 \%)$ & 4438 (98.4\%) & $3530(98.0 \%)$ & 0.38 \\
\hline \multicolumn{7}{|l|}{ Race } \\
\hline White & $13,613(86.5 \%)$ & $2997(85.5 \%)$ & $3525(85.8 \%)$ & 3919 (86.9\%) & $3172(88.0 \%)$ & $<.0001$ \\
\hline Black & 1799 (11.4\%) & $421(12.0 \%)$ & $525(12.8 \%)$ & $515(11.4 \%)$ & $338(9.4 \%)$ & \\
\hline Other & $317(2.0 \%)$ & $89(2.5 \%)$ & $58(1.4 \%)$ & 77 (1.7\%) & $93(2.6 \%)$ & \\
\hline \multicolumn{7}{|l|}{ Cormorbidities } \\
\hline Hypertension & $14,973(95.2 \%)$ & 3332 (95.0\%) & $3953(96.2 \%)$ & $4242(94.0 \%)$ & $3446(95.6 \%)$ & $<.0001$ \\
\hline Hyperlipidemia & $14,413(91.6 \%)$ & $3146(89.7 \%)$ & $3855(93.8 \%)$ & $4077(90.4 \%)$ & $3335(92.6 \%)$ & $<.0001$ \\
\hline Diabetes & $9127(58.0 \%)$ & 2060 (58.7\%) & $2407(58.6 \%)$ & $2552(56.6 \%)$ & $2108(58.5 \%)$ & 0.14 \\
\hline Tobacco Use & $9124(58.0 \%)$ & $1916(54.6 \%)$ & $2599(63.3 \%)$ & 2339 (51.9\%) & $2270(63.0 \%)$ & $<.0001$ \\
\hline Prior Ml & $6728(42.8 \%)$ & 1503 (42.9\%) & $1804(43.9 \%)$ & $1860(41.2 \%)$ & $1561(43.3 \%)$ & 0.071 \\
\hline Prior PCl & 6018 (38.3\%) & 1328 (37.9\%) & $1602(39.0 \%)$ & 1743 (38.6\%) & $1345(37.3 \%)$ & 0.43 \\
\hline Congestive Heart Failure & $5893(37.5 \%)$ & $1330(37.9 \%)$ & $1503(36.6 \%)$ & $1711(37.9 \%)$ & $1349(37.4 \%)$ & 0.56 \\
\hline Cerebrovascular Disease & $3803(24.2 \%)$ & $881(25.1 \%)$ & $982(23.9 \%)$ & $1034(22.9 \%)$ & $906(25.1 \%)$ & 0.055 \\
\hline Peripheral Arterial Disease & $4451(28.3 \%)$ & $970(27.7 \%)$ & $1152(28.0 \%)$ & $1220(27.0 \%)$ & $1109(30.8 \%)$ & 0.0016 \\
\hline Hx of CKD Dx & 8707 (55.4\%) & $1876(53.5 \%)$ & $2319(56.5 \%)$ & $2308(51.2 \%)$ & $2204(61.2 \%)$ & $<.0001$ \\
\hline $\begin{array}{l}\text { Chronic Obstructive Pulmonary } \\
\text { Disease }\end{array}$ & $4136(26.3 \%)$ & $930(26.5 \%)$ & $1035(25.2 \%)$ & $1229(27.2 \%)$ & 942 (26.1\%) & 0.19 \\
\hline BMI (Median (IQR)) & $29.9(26.4-34.0)$ & $30.0(26.6-34.1)$ & $30.1(26.6-34.2)$ & $29.7(26.1-33.7)$ & $29.9(26.4-34.1)$ & 0.0048 \\
\hline Chronic Depression & 4407 (28.0\%) & $1004(28.6 \%)$ & $1263(30.7 \%)$ & $1213(26.9 \%)$ & 927 (25.7\%) & $<.0001$ \\
\hline Sleep Apnea & $3596(22.9 \%)$ & $765(21.8 \%)$ & $1008(24.5 \%)$ & $993(22.0 \%)$ & $830(23.0 \%)$ & 0.014 \\
\hline Baseline Cholesterol (Median (IQR)) & $\begin{array}{l}157.7(135.3- \\
185.0)\end{array}$ & $\begin{array}{l}161.5(138.0- \\
189.0)\end{array}$ & $\begin{array}{l}160.1(137.2- \\
188.0)\end{array}$ & $\begin{array}{l}155.2(133.0- \\
183.0)\end{array}$ & $\begin{array}{l}154.0(133.0- \\
181.0)\end{array}$ & $<.0001$ \\
\hline Baseline LDL (Median (IQR)) & $85.8(67.7-108.4)$ & $86.3(68.3-110.0)$ & $87.1(68.0-109.2)$ & $86.0(68.0-108.3)$ & $83.3(66.0-106.0)$ & $<.0001$ \\
\hline Baseline HDL (Median (IQR)) & $36.7(31.0-43.5)$ & $36.6(31.0-43.6)$ & $36.5(31.0-43.0)$ & $36.3(31.0-43.0)$ & $37.1(31.7-44.3)$ & 0.0006 \\
\hline \multicolumn{7}{|l|}{ CKD class } \\
\hline $3 a$ & $11,029(70.1 \%)$ & $2404(68.5 \%)$ & $2958(72.0 \%)$ & $3140(69.6 \%)$ & $2527(70.1 \%)$ & 0.02 \\
\hline $3 b$ & 3927 (25.0\%) & 915 (26.1\%) & $981(23.9 \%)$ & $1135(25.2 \%)$ & $896(24.9 \%)$ & \\
\hline 4 & $773(4.9 \%)$ & $188(5.4 \%)$ & $169(4.1 \%)$ & $236(5.2 \%)$ & $180(5.0 \%)$ & \\
\hline Baseline GFR (Median (IQR)) & $50.4(43.0-55.7)$ & $50.0(42.4-55.1)$ & $50.9(43.8-56.0)$ & $50.6(43.0-56.0)$ & $50.2(42.9-55.3)$ & 0.0012 \\
\hline \multicolumn{7}{|l|}{ Procedural Details } \\
\hline \multicolumn{7}{|l|}{ PCI Status } \\
\hline Elective & 9617 (61.1\%) & $2275(64.9 \%)$ & $2744(66.8 \%)$ & $2545(56.4 \%)$ & $2053(57.0 \%)$ & $<.0001$ \\
\hline Emergent/Urgent & $6034(38.4 \%)$ & $1220(34.8 \%)$ & $1329(32.4 \%)$ & $1947(43.2 \%)$ & $1538(42.7 \%)$ & \\
\hline Missing & $78(0.50 \%)$ & $12(0.3 \%)$ & $35(0.9 \%)$ & $19(0.4 \%)$ & $12(0.3 \%)$ & \\
\hline \multicolumn{7}{|l|}{ PCl Indication } \\
\hline STEMI & $1094(7.0 \%)$ & $263(7.5 \%)$ & $252(6.1 \%)$ & $336(7.4 \%)$ & $243(6.7 \%)$ & $<.00$ \\
\hline NSTEMI & 3515 (22.3\%) & $756(21.6 \%)$ & 789 (19.2\%) & $1074(23.8 \%)$ & 896 (24.9\%) & \\
\hline Unstable Angina & 3516 (22.4\%) & $682(19.4 \%)$ & 1154 (28.1\%) & $918(20.4 \%)$ & 762 (21.1\%) & \\
\hline ACS & $182(1.2 \%)$ & 39 (1.1\%) & 44 (1.1\%) & 74 (1.6\%) & $25(0.7 \%)$ & \\
\hline Stable Angina & 4693 (29.8\%) & $1218(34.7 \%)$ & 1119 (27.2\%) & $1285(28.5 \%)$ & 1071 (29.7\%) & \\
\hline
\end{tabular}


Table 1 Demographic and clinical characteristics of patients with CKD receiving PCI arranged by site quartile of unadjusted rates of periprocedural hydration or NAC use (Continued)

\begin{tabular}{|c|c|c|c|c|c|c|}
\hline Variable & $\begin{array}{l}\text { Total } \\
(N=15,729)\end{array}$ & $\begin{array}{l}\text { QUARTILE } 1 \\
(N=3507)\end{array}$ & $\begin{array}{l}\text { QUARTILE } 2 \\
(N=4108)\end{array}$ & $\begin{array}{l}\text { QUARTILE } 3 \\
(N=4511)\end{array}$ & $\begin{array}{l}\text { QUARTILE } 4 \\
(N=3603)\end{array}$ & $p$-value \\
\hline Other/Missing & $2729(17.4 \%)$ & $549(15.7 \%)$ & $750(18.3 \%)$ & $824(18.3 \%)$ & $606(16.8 \%)$ & \\
\hline At least 1 lesion of high risk & $6586(41.9 \%)$ & $1578(45.0 \%)$ & $1535(37.4 \%)$ & $2056(45.6 \%)$ & 1417 (39.3\%) & $<.0001$ \\
\hline Number of stents (Median (IQR)) & $1.0(1.0-1.0)$ & $1.0(1.0-1.0)$ & $1.0(1.0-1.0)$ & $1.0(1.0-1.0)$ & $1.0(1.0-1.0)$ & 0.64 \\
\hline \multicolumn{7}{|l|}{ CA-AKI Prevention Measures } \\
\hline \multicolumn{7}{|c|}{ Mutually Exclusive Categories of Preventions } \\
\hline None & $6293(40.0 \%)$ & $2028(57.8 \%)$ & $1778(43.3 \%)$ & $1537(34.1 \%)$ & $950(26.4 \%)$ & \multirow[t]{4}{*}{$<.0001$} \\
\hline Only NS & $4329(27.5 \%)$ & $533(15.2 \%)$ & $1004(24.4 \%)$ & $1464(32.5 \%)$ & 1328 (36.9\%) & \\
\hline Only NAC & $2508(15.9 \%)$ & $691(19.7 \%)$ & $604(14.7 \%)$ & $572(12.7 \%)$ & $641(17.8 \%)$ & \\
\hline NAC and Saline & $2599(16.5 \%)$ & $255(7.3 \%)$ & $722(17.6 \%)$ & $938(20.8 \%)$ & $684(19.0 \%)$ & \\
\hline Any NAC & 5107 (32.5\%) & $946(27.0 \%)$ & $1326(32.3 \%)$ & $1510(33.5 \%)$ & 1325 (36.8\%) & $<.0001$ \\
\hline Any Hydration & 6928 (44.0\%) & $788(22.5 \%)$ & $1726(42.0 \%)$ & $2402(53.2 \%)$ & $2012(55.8 \%)$ & $<.0001$ \\
\hline Hydration or NAC & $9436(60.0 \%)$ & 1479 (42.2\%) & $2330(56.7 \%)$ & $2974(65.9 \%)$ & $2653(73.6 \%)$ & $<.0001$ \\
\hline Contrast use & $(N=12,938)$ & $(N=2988)$ & $(N=3096)$ & $(N=3720)$ & $(N=3134)$ & \\
\hline Contrast (Median (IQR)) & $\begin{array}{l}175.0(120.0- \\
246.0)\end{array}$ & $\begin{array}{l}165.0(100.0- \\
230.0)\end{array}$ & $\begin{array}{l}175.0(120.0- \\
240.0)\end{array}$ & $\begin{array}{l}190.0(132.0- \\
260.0)\end{array}$ & $\begin{array}{l}165.0(105.0- \\
235.0)\end{array}$ & $<.0001$ \\
\hline Contrast:GFR (Median (IQR)) & $3.6(2.5-5.1)$ & $3.4(2.2-5.0)$ & $3.6(2.5-5.1)$ & $4.0(2.8-5.4)$ & $3.5(2.3-4.9)$ & $<.0001$ \\
\hline Contrast:GFR $\leq 3$ & $4656(36.0 \%)$ & 1206 (40.4\%) & 1136 (36.7\%) & 1081 (29.1\%) & 1233 (39.3\%) & $<.0001$ \\
\hline
\end{tabular}

To our knowledge, only a small number of studies have investigated the prevalence of CA-AKI prophylactic measure utilization, none of which analyzed contrast minimization strategies, or PCI and CKD specifically [21-24]. A 2008 single-center study by Weisbord et al. prospectively identified 660 patients with CKD undergoing intravenous and intra-arterial contrasted studies and showed that periprocedural fluids were administered in $40 \%$ of patients and NAC was administered in $39.2 \%$ [23]. A 2014 study by Lee et al. retrospectively analyzed the incidence of CA-AKI and prevalence of prophylactic measure utilization with intravenous contrasted computed tomography (CT) scans. They identified 101,487 patients with 140,838 contrasted CT scans and found the incidence of prophylactic medication utilization to be $28.6 \%$, mostly driven by NS use (26\%) [24].

Table 2 Risk-Adjusted Median Odds Ratio for CA-AKI prophylaxis measures in $\mathrm{PCl}$ patients with CKD and all $\mathrm{PCl}$ patients (CKD or normal kidney function)

\begin{tabular}{lllllll}
\hline & \multicolumn{2}{l}{ CKD Patients } & & \multicolumn{2}{l}{ All Patients } \\
\cline { 2 - 3 } & MOR & $95 \% \mathrm{Cl}$ & & MOR & $95 \% \mathrm{Cl}$ \\
\hline Hydration & 1.80 & $1.59-2.08$ & & 1.96 & $1.71-2.24$ \\
Hydration or NAC & 1.95 & $1.66-2.29$ & & 2.15 & $1.82-2.53$ \\
Contrast:GFR Ratio $\leq 3$ & 2.68 & $2.23-3.15$ & & 2.75 & $2.26-3.28$ \\
\hline
\end{tabular}

Estimates risk-adjusted for the following: $\mathrm{CHF}$, age, sex, race (white, black, other), GFR, diabetes, lesion risk (at least 1 high vs all non-high), \# of stents, year of $\mathrm{PCl}$, and annual hospital $\mathrm{PCl}$ volume
Compared to these, our study specifically analyzed the prevalence of CA-AKI prophylaxis surrounding PCI in patients with and without CKD, including contrast minimization. Our finding of periprocedural hydration or NAC administration in $43 \%$ of cases (patients with and without CKD) is within range of prior literature. In addition, we add to this literature with our demonstration of a high level of patient-adjusted variability in CAAKI prophylaxis among high-risk patients, including contrast minimization, and accounting for patient-level risk factors.

The significant variation in CA-AKI prophylaxis likely reflects the ambiguity of the underlying data, particularly in light of recent randomized clinical trials. A study by Nijssen et al. demonstrated no benefit when comparing periprocedural hydration with NS to no intravenous hydration at all [25]. The PRESERVE trial randomly assigned 5177 patients to receive periprocedural hydration with intravenous sodium bicarbonate or NS, as well as either NAC or placebo, in a 2-by-2 factorial design. The study demonstrated no benefit of sodium bicarbonate compared to NS, as well as no benefit of NAC when compared to placebo [26].

Conflicting major society guidelines likely compound the effect of the contradictory literature. The 2011 ACCF/AHA/SCAI guidelines for PCI recommend periprocedural intravenous hydration without a 


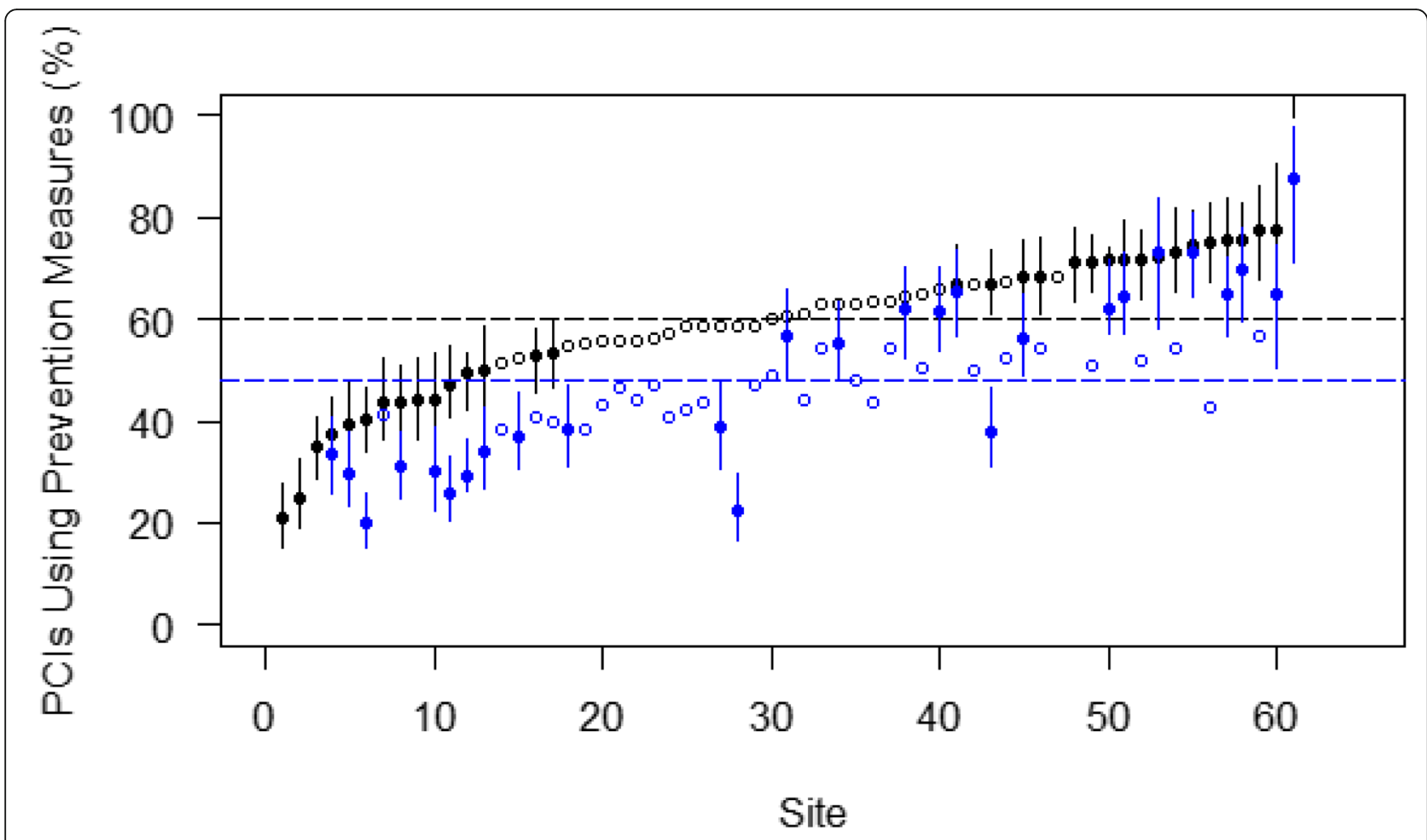

\section{Hydration or NAC Hydration \\ $\rightarrow$ Differs from overall mean (with $95 \% \mathrm{Cl}$ ) \\ - Does not differ significantly}

Fig. 2 Plot of risk-adjusted, site-level rates of hydration and NAC prophylaxis in PCI patients with CKD. The estimates are shown with 95\% confidence intervals $(\mathrm{Cls})$ for those that differ significantly from the system-wide average. Estimates are risk-adjusted for the following: $\mathrm{CHF}$, age, sex, race (white, black, other), GFR, diabetes, lesion risk (at least 1 high vs all non-high), number of stents, year of $\mathrm{PCl}$, and annual hospital $\mathrm{PCl}$ volume

specific protocol for volume or timing of hydration and recommend against NAC [12]. The 2012 KDIGO guidelines recommend periprocedural intravenous hydration without specific protocols for volume or timing of hydration, however they do recommend the use of NAC [13]. Accordingly, this inconsistency in the hydration and NAC guidelines makes our findings of high variability in their utilization somewhat expected.

In contrast to these inconsistencies, there is broad consensus and guideline support for minimizing contrast during PCI, and numerous studies have demonstrated a contrast: GFR volume $<3$ is associated with lower rates of CA-AKI [10]. This makes our findings of high variability in contrast minimization, both in our overall population and among those with CKD, very surprising.

Our study had several limitations, the first being that our outcomes were determined from analyzing the electronic health record, and some data may have been incompletely or inaccurately recorded, with accuracy varying by site. This could have resulted in inaccurate measurement of prophylactic measures. Second, the results may be subject to confounding variables not accounted for by our statistical models. We adjusted for numerous variables associated with CA-AKI, however, there could be unaccounted for confounders that influenced our results. Third, we used an arbitrary volume of $>100 \mathrm{~mL}$ periprocedural hydration as a cutoff for receiving prophylactic hydration. However, the most commonly used NS hydration protocols $(3-4 \mathrm{~mL} / \mathrm{kg}$ per hour $4 \mathrm{~h}$ before and $4 \mathrm{~h}$ after contrast administration, or $1 \mathrm{~mL} / \mathrm{kg}$ per hour $12 \mathrm{~h}$ before and $12 \mathrm{~h}$ after) would administer $1.5-2.01$ of NS for a typical $70 \mathrm{~kg}$ patient; thus a cutoff of $100 \mathrm{~mL}$ of NS likely over estimates the rate of prophylactic NS administration [25]. Fourth, there are numerous other CA-AKI prophylactic strategies 


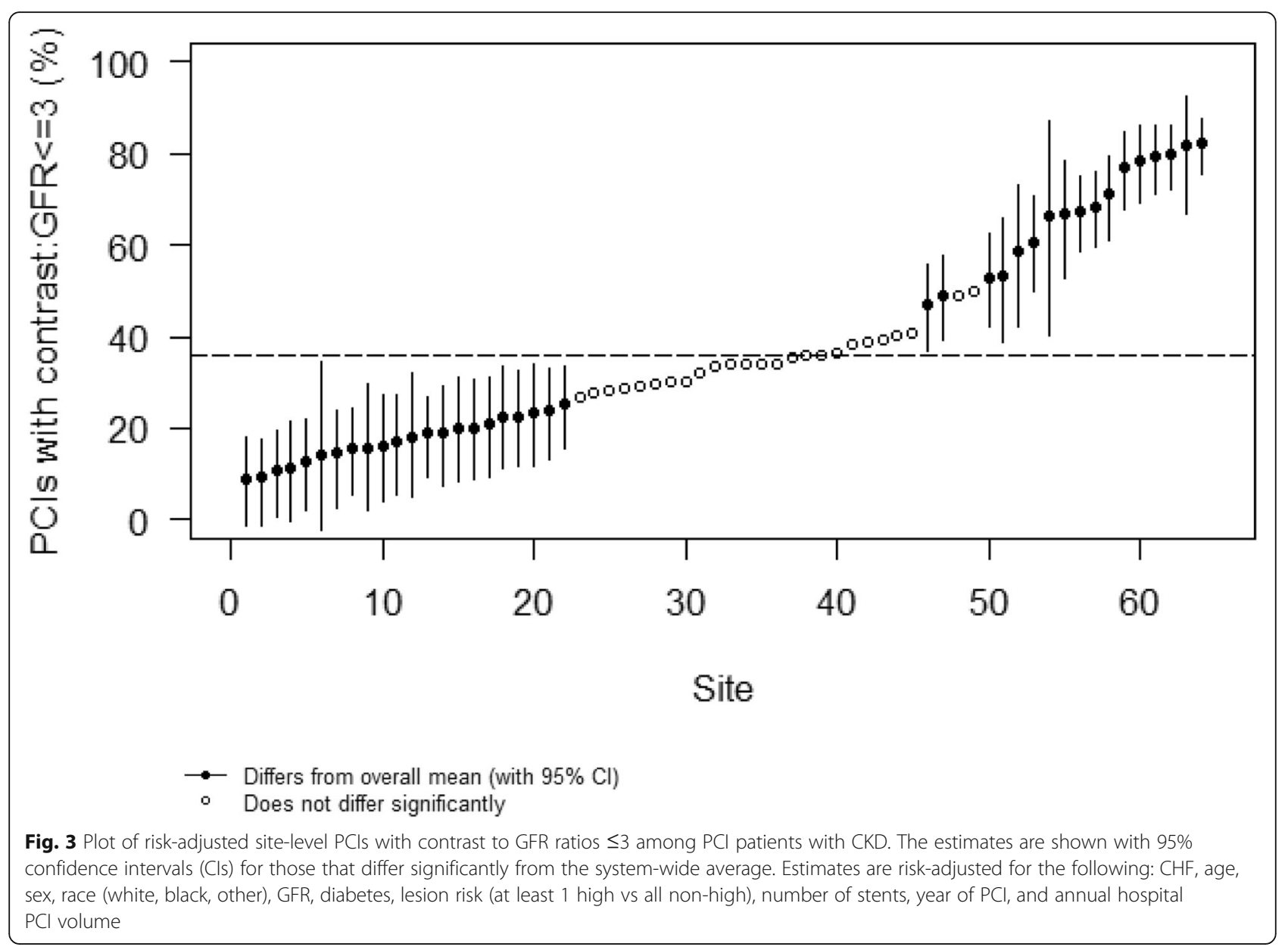

available, including statin therapy and ascorbic acid, which were not evaluated in this study. However, as periprocedural hydration, NAC, and contrast minimization have the strongest body of evidence supporting their use, we believe they are adequate surrogates. Fifth, there is ongoing debate in the literature on the prevalence and nature of CA-AKI, with some evidence suggesting the prevalence is lower than originally thought [27]. This underlying confusion, however, likely leads to some of the variation we identified, and until this condition is better understood practice variation will continue. Sixth, we did not capture whether certain nephrotoxic home medications were held or continued (diuretics, NSAIDs, ACE/ARB/RAAS inhibitors, etc.). This could have potentially informed our investigation further on CA-AKI prophylactic provider behaviors. Seventh, we did not adjust for elective vs. urgent/emergent procedure type, nor the presence or absence of cardiogenic shock. We did, however, adjust for the presence or absence of CHF as well as lesion risk and number of stents, which we felt were adequate surrogates. Eighth, we acknowledge that contrast minimization may not have been possible in certain cases and variability in this may not necessarily reflect provider non-use of the intervention.

\section{Conclusion}

In conclusion, our study demonstrated a low overall prevalence of CA-AKI prophylaxis, and a high level of site variation in VA catheterization laboratories for both patients with CKD and the overall PCI population. This variability was present with both established methods, such as contrast minimization, and contestable methods, such as hydration or NAC administration. There are likely multiple factors causing this variability, including conflicting literature, vague and contradictory guidelines, and provider preference. Proven methods to prevent CA-AKI, such as contrast minimization, should be standardized across the healthcare system, while further research should be pursued to establish other effective measures to prevent CA-AKI, and prospectively evaluate the influence of standardized CA-AKI prophylaxis protocols on patient outcomes. 


\section{Supplementary information}

Supplementary information accompanies this paper at https://doi.org/10. 1186/s12882-020-01802-z.

Additional file 1: Table S1a, b, and c Temporal Trends in CA-AKI prophylaxis utilization

Additional file 2: Figure S1 Plot of risk-adjusted, site-level rates of hydration and NAC prophylaxis among all PCl patients (with and without CKD). The estimates are shown with 95\% confidence intervals (Cls) for those that differ significantly from the system-wide average. Estimates are risk-adjusted for the following: CHF, age, sex, race (white, black, other), GFR, diabetes, lesion risk (at least 1 high vs all non-high), number of stents, year of $\mathrm{PCl}$, and annual hospital $\mathrm{PCl}$ volume. Figure $\mathbf{S 2}$ Plot of risk-adjusted, site-level $\mathrm{PCl}$ s with contrast to GFR ratios $\leq 3$ among all $\mathrm{PCl}$ patients (with and without CKD). The estimates are shown with 95\% confidence intervals (Cls) for those that differ significantly from the systemwide average. Estimates are risk-adjusted for the following: CHF, age, sex, race (white, black, other), GFR, diabetes, lesion risk (at least 1 high vs all non-high), number of stents, year of $\mathrm{PCl}$, and annual hospital $\mathrm{PCl}$ volume. Figure S3 Plot of site-level saline use among CKD patients, stratified by CHF. The estimates are shown with 95\% confidence intervals (Cls) for those that differ significantly from the system-wide average. Estimates are risk-adjusted for the following: age, sex, race (white, black, other), GFR, diabetes, lesion risk (at least 1 high vs all non-high), number of stents, year of $\mathrm{PCl}$, and annual hospital $\mathrm{PCl}$ volume.

\section{Abbreviations}

CA-AKI: Contrast-Associated Acute Kidney Injury; GFR: glomerular filtration rate; $\mathrm{PCl}$ : percutaneous coronary intervention; NAC: N-Acetylcysteine; CKD: chronic kidney disease; MOR: median odds ratio

\section{Acknowledgements}

Not applicable.

\section{Other disclosures}

The content of this article does not necessarily reflect the views of the U. S, Government or the Department of Veteran's Affairs.

\section{Authors' contributions}

All authors (JK, MS, AB, MP, PL, AA, HG, SW, TM) read and approved the final manuscript. All authors (JK, MS, AB, MP, PL, AA, HG, SW, TM) participated in the interpretation of the data and writing of the manuscript. MS, MP, and $A B$ performed the primary analysis of the data. The author(s) read and approved the final manuscript.

\section{Funding}

No funding source has any input into the design of the study, nor the collection, analysis, and interpretation of data, nor in writing the manuscript.

\section{Availability of data and materials}

The datasets used and/or analyzed during the current study are available from the corresponding author on reasonable request.

\section{Ethics approval and consent to participate}

The Colorado Multiple Institutional Review Board approved the study.

\section{Consent for publication}

Not applicable.

\section{Competing interests}

The authors declare that they have no competing interests.

\section{Author details}

'Department of Medicine, Denver Health Hospital Authority, 601 Broadway MC4000, Denver, CO 80204, USA. ²Department of Medicine, University of Colorado, Aurora, CO, USA. ${ }^{3}$ Rocky Mountain Regional VA Medical Center, Aurora, CO, USA. ${ }^{4}$ Colorado School of Public Health, University of Colorado, Aurora, CO, USA. ${ }^{5}$ Division of Cardiology, Washington University School of Medicine, St. Louis, MO, USA. ${ }^{6}$ Healthcare Innovation Lab, BJC HealthCare/ Washington University School of Medicine, St. Louis, MO, USA.
Received: 3 November 2019 Accepted: 12 April 2020

Published online: 28 April 2020

\section{References}

1. Parsh J, Seth M, Briguori C, Grossman P, Solomon R, Gurm HS. The optimal definition of contrast-induced acute kidney injury for prediction of inpatient mortality in patients undergoing percutaneous coronary interventions. Am Heart J. 2016;175:160-7.

2. Rihal CS, Textor SC, Grill DE, Berger PB, Ting HH, Best PJ, et al. Incidence and prognostic importance of acute renal failure after percutaneous coronary intervention. Circulation. 2002;105(19):2259-64.

3. Subramanian S, Tumlin J, Bapat B, Zyczynski T. Economic burden of contrast-induced nephropathy: implications for prevention strategies. J Med Econ. 2007;10(2):119-34.

4. Mitchell $A M$, Jones AE, Tumlin JA, Kline JA. Incidence of contrast-induced nephropathy after contrast-enhanced computed tomography in the outpatient setting. Clin J Am Soc Nephrol. 2010;5(1):4-9.

5. Weisbord SD, Chen H, Stone RA, Kip KE, Fine MJ, Saul Ml, et al. Associations of increases in serum creatinine with mortality and length of hospital stay after coronary angiography. J Am Soc Nephrol. 2006;17(10):2871-7.

6. Tsai TT, Patel UD, Chang TI, Kennedy KF, Masoudi FA, Matheny ME, et al. Contemporary incidence, predictors, and outcomes of acute kidney injury in patients undergoing percutaneous coronary interventions: insights from the NCDR Cath-PCI registry. JACC Cardiovasc Interv. 2014;7(1):1-9.

7. Subramaniam RM, Suarez-Cuervo C, Wilson RF, Turban S, Zhang A, Sherrod $C$, et al. Effectiveness of prevention strategies for contrast-induced nephropathy: a systematic review and meta-analysis. Ann Intern Med. 2016; 164(6):406-16.

8. McCullough PA, Choi JP, Feghali GA, Schussler JM, Stoler RM, Vallabahn RC, et al. Contrast-induced acute kidney injury. J Am Coll Cardiol. 2016;68(13): 1465-73.

9. McCullough PA, Wolyn R, Rocher LL, Levin RN, O'Neill WW. Acute renal failure after coronary intervention: incidence, risk factors, and relationship to mortality. Am J Med. 1997;103(5):368-75.

10. Gurm HS, Dixon SR, Smith DE, Share D, Lalonde T, Greenbaum A, et al. Renal function-based contrast dosing to define safe limits of radiographic contrast media in patients undergoing percutaneous coronary interventions. J Am Coll Cardiol. 2011;58(9):907-14.

11. Mueller C, Buerkle G, Buettner HJ, Petersen J, Perruchoud AP, Eriksson U, et al. Prevention of contrast media-associated nephropathy: randomized comparison of 2 hydration regimens in 1620 patients undergoing coronary angioplasty. Arch Intern Med. 2002;162(3):329-36.

12. Levine GN, Bates ER, Blankenship JC, Bailey SR, Bittl JA, Cercek B, et al. 2011 ACCF/AHA/SCAI guideline for percutaneous coronary intervention: a report of the American College of Cardiology Foundation/American Heart Association task force on practice guidelines and the Society for Cardiovascular Angiography and Interventions. Circulation. 2011;124(23): e574-651.

13. Khwaja A. KDIGO clinical practice guidelines for acute kidney injury. Nephron Clin Pract. 2012;120(4):c179-84.

14. Maddox TM, Plomondon ME, Petrich M, Tsai TT, Gethoffer H, Noonan G, et al. A national clinical quality program for veterans affairs catheterization laboratories (from the veterans affairs clinical assessment, reporting, and tracking program). Am J Cardiol. 2014;114(11):1750-7.

15. Brindis RG, Fitzgerald S, Anderson HV, Shaw RE, Weintraub WS, Williams JF. The American College of Cardiology-National Cardiovascular Data Registry (ACC-NCDR): building a national clinical data repository. J Am Coll Cardiol. 2001;37(8):2240-5

16. Byrd JB, Vigen R, Plomondon ME, Rumsfeld JS, Box TL, Fihn SD, et al. Data quality of an electronic health record tool to support VA cardiac catheterization laboratory quality improvement: the VA clinical assessment, reporting, and tracking system for Cath labs (CART) program. Am Heart J. 2013;165(3):434-40.

17. Levey AS, Bosch JP, Lewis JB, Greene T, Rogers N, Roth D. A more accurate method to estimate glomerular filtration rate from serum creatinine: a new prediction equation. Modification of diet in renal disease study group. Ann Intern Med. 1999;130(6):461-70

18. Leora Horwitz M, MHS. Hospital-Wide All-Cause Unplanned Readmission Measure Final Technical Report 2012 [updated 09/07/2017 1:06 PM. Available from: https://www.cms.gov/Medicare/Quality-Initiatives-PatientAssessment-Instruments/HospitalQualitylnits/Measure-Methodology.html. 
19. Timbie JW, Normand SL. A comparison of methods for combining quality and efficiency performance measures: profiling the value of hospital care following acute myocardial infarction. Stat Med. 2008;27(9):1351-70.

20. Chan PS, Maddox TM, Tang F, Spinler S, Spertus JA. Practice-level variation in warfarin use among outpatients with atrial fibrillation (from the NCDR PINNACLE program). Am J Cardiol. 2011;108(8):1136-40.

21. Weisbord SD, Bruns FJ, Saul MI, Palevsky PM. Provider use of preventive strategies for radiocontrast nephropathy in high-risk patients. Nephron Clin Pract. 2004;96(2):c56-62.

22. Weisbord SD, Mor MK, Kim S, Hartwig KC, Sonel AF, Palevsky PM, et al. Factors associated with the use of preventive care for contrast-induced acute kidney injury. J Gen Intern Med. 2009;24(3):289-98.

23. Weisbord SD, Mor MK, Resnick AL, Hartwig KC, Sonel AF, Fine MJ, et al. Prevention, incidence, and outcomes of contrast-induced acute kidney injury. Arch Intern Med. 2008;168(12):1325-32.

24. Lee J, Cho JY, Lee HJ, Jeong YY, Kim CK, Park BK, et al. Contrast-induced nephropathy in patients undergoing intravenous contrast-enhanced computed tomography in Korea: a multi-institutional study in 101487 patients. Korean J Radiol. 2014;15(4):456-63.

25. Nijssen EC, Rennenberg RJ, Nelemans PJ, Essers BA, Janssen MM, Vermeeren $M A$, et al. Prophylactic hydration to protect renal function from intravascular iodinated contrast material in patients at high risk of contrast-induced nephropathy (AMACING): a prospective, randomised, phase 3, controlled, open-label, non-inferiority trial. Lancet. 2017;389(10076):1312-22.

26. Weisbord SD, Gallagher M, Jneid H, Garcia S, Cass A, Thwin S-S, et al. Outcomes after Angiography with Sodium Bicarbonate and Acetylcysteine. http://dxdoiorg/101056/NEJMoa1710933. 2017

27. Wilhelm-Leen E, Montez-Rath ME, Chertow G. Estimating the risk of Radiocontrast-associated nephropathy. J Am Soc Nephrol. 2017;28(2):653-9.

\section{Publisher's Note}

Springer Nature remains neutral with regard to jurisdictional claims in published maps and institutional affiliations.

Ready to submit your research? Choose BMC and benefit from:

- fast, convenient online submission

- thorough peer review by experienced researchers in your field

- rapid publication on acceptance

- support for research data, including large and complex data types

- gold Open Access which fosters wider collaboration and increased citations

- maximum visibility for your research: over $100 \mathrm{M}$ website views per year

At $\mathrm{BMC}$, research is always in progress.

Learn more biomedcentral.com/submissions 